\title{
Double Parton Scattering in Associate Higgs Boson Production with Heavy Quarks at the LHC
}

\author{
Mohammad Yousif Hussein \\ Physics Department, College of Science, Bahrain University, Sakhir, Kingdom of Bahrain \\ Email: mhussein@sci.uob.bh
}

Received February 6, 2013; revised March 8, 2013; accepted March 20, 2013

Copyright (C) 2013 Mohammad Yousif Hussein. This is an open access article distributed under the Creative Commons Attribution License, which permits unrestricted use, distribution, and reproduction in any medium, provided the original work is properly cited.

\begin{abstract}
Higgs boson production in association with heavy quarks is one of the most important discovery channels for Higgs particles in the Standard Model and its super-symmetric extension at the LHC $p p$ collider. We review the status of the Higgs boson studies, with particular emphasis on the case $b \bar{b} h$ and $t \overline{t h}$ production. We present results for the total cross section at Large Hadron Collider (LHC) in both single and double parton scattering mechanism.
\end{abstract}

Keywords: Standard Model; Higgs Boson; Double Parton Scattering

\section{Introduction}

In the simplest version of the Standard Model (SM) of particle physics, the breaking of the electroweak symmetry introduces a single physical scalar particle, the Higgs boson, which couples to both gauge boson and fermions. Extensions of the Standard Model, like the Minimal Supersymmetric Standard (MSSM), introduce several scalar and pseudoscalar Higgs boson.

Much progress has been made in the detection of a Higgs boson, next-leading order corrections are now known for most sub-processes [1-8], and knowledge of parton distribution functions has improved as more deep inelastic data become available and the range of possible input parameter values decreased.

Recently, ATLAS [9] and CMS [10] collaborations announced about the discovery of a new scalar particle at the Large Hadron Collider (LHC), which is most likely the Standard Model (SM) Higgs boson, with the mass measured $m_{h}=125.3 \pm 0.4 \mathrm{GeV}$.

Although we need more data accumulation to conclude it is truly the SM Higgs boson, these observations have ignited a new trend of particle physics research.

After the discovery of the Higgs boson at the LHC, it would be important to study various properties of it. In particular, one would like to study the production of the Higgs boson via all possible channels. One such category of channels is the production of the Higgs boson in association with heavy quarks.

The associated production of a Higgs boson with a $t \bar{t}$ air at the LHC, $p p \rightarrow \overline{t t h}$, will play a very important role in the Higgs boson mass range, both for discovery and for precision measurements of the Higgs boson couplings. This process will provide a direct measurement of the top quark Yukawa coupling which could help to distinguish a SM Higgs boson from more complex Higgs sector and shed light on the details of the generation of fermions masses [11-17].

Although the associated production of a Higgs boson with a pair of $b \bar{b}$ quarks has a small cross section in the SM, but it can therefore be used to test the hypothesis of enhanced bottom quark Yukawa coupling which is common to many extensions of the SM, such as the MSSM.

The coupling of the Higgs boson to $b \bar{b}$ pair is suppressed in the Standard Model by the small factor, $m_{b} / v$, where $v=\left(\sqrt{2} G_{F}\right)^{-1 / 2}=246 \mathrm{GeV}$.

In this context, we focus on the production of a Higgs boson with a pair of top or bottom quark and anti-quark. Our calculation corresponds to the inclusive cross section for Higgs boson production in association with heavy quarks, integrated over the momenta of $t$ and $b$ quarks.

When looking at extrapolation of the cross section at high energies, one finds that the results are affected by several uncertainties, as the knowledge of the parton structure functions at very small $x$ and the values of the heavy quark mass and of the running coupling constant. Although the expected inclusive cross section of $b$ 
production is hence still pretty uncertain at LHC energy, all estimates point in the direction of rather large values, as a consequence of the high parton luminosity. The fairly large flux of partons makes it also plausible to expect a sizable rate of events, where two $b \bar{b}$ pair with Higgs boson is produced contemporarily by different partonic collisions in a given $p p$ interaction. Although at present stage all quantitative prediction for this much more structured interaction process are unavoidably pretty uncertain, the large cross sections foreseen at the LHC is, in our opinion, a strong motivation to make an attempt of giving a few quantitative indications on the production rate of associated Higgs with heavy quark pairs through multiparton interaction at the LHC, comparing with the rate to be expected by the more conventional single parton scattering mechanism.

\section{Double Parton Scattering Mechanism}

The multiple parton scattering occurs when two or more different pairs of parton scatter independently in the same hadronic collision [8-23]. A schematic view of a double parton scattering events in a $p^{\bar{p}}$ interaction is shown in Figure 1.

With the only assumption of factorization of the two hard parton processes $A$ and $B$, the inclusive cross section of a double parton-scattering in a hadronic collision is expressed by:

$$
\begin{aligned}
\sigma_{(A, B)}^{D}= & \frac{m}{2} \sum_{i, j, k, l} \Gamma_{i, j}\left(x_{1}, x_{2} ; b\right) \hat{\sigma}_{i k}^{A}\left(x_{1}, x_{1}^{\prime}\right) \\
& \cdot \hat{\sigma}_{j l}^{B}\left(x_{2}, x_{2}^{\prime}\right) \Gamma_{k, l}\left(x_{1}^{\prime}, x_{2}^{\prime} ; b\right) \mathrm{d} x_{1} \mathrm{~d} x_{1}^{\prime} \mathrm{d} x_{2} \mathrm{~d} x_{2}^{\prime} \mathrm{d}^{2} b,
\end{aligned}
$$

where $\Gamma_{i j}\left(x_{1}, x_{2} ; b\right)$ are the double parton distribution function, depending on the fractional momenta $x_{1}, x_{2}$ and the relative transverse distance $b$ of the two parton undergoing the hard processes $A$ and $B$, the indices $i$ and $j$ refer to the different parton species and $\hat{\sigma}_{j l}^{B}$ and $\hat{\sigma}_{i k}^{A}$ are the partonic cross section. The factor $m / 2$ is for symmetry, specifically $m=1$ is for indistinguishable parton processes and $m=2$ is for distinguishable

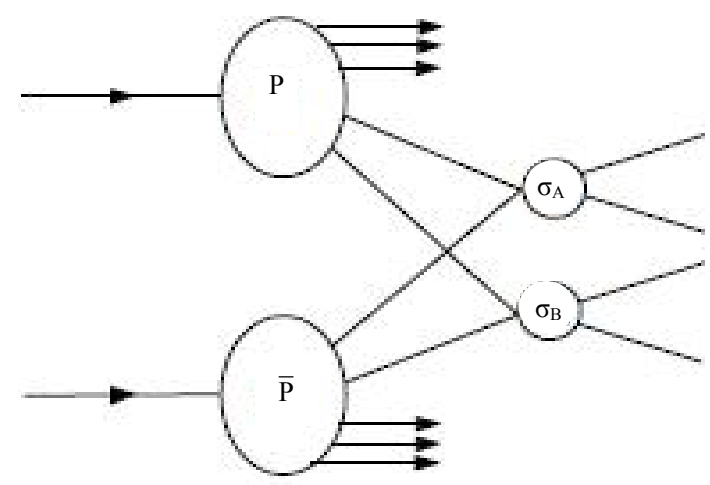

Figure 1. Diagram of a double parton scattering. processes.

The double distributions $\Gamma_{i j}\left(x_{1}, x_{2} ; b\right)$ are the main reason of interest in multiparton collisions. This distribution contains in fact all the information of probing the hadron in two different points contemporarily through the hard processes $A$ and $B$.

The cross section for multiparton process is sizable when the flux of partons is large, namely at small $x$. Given the large flux one may hence expect that correlations in momentum fraction will not be a major effect and partons to be rather correlated in transverse space. Neglecting the effect of parton correlations in $x$ one writes:

$$
\Gamma_{i j}\left(x_{1}, x_{2} ; b\right)=\Gamma_{i}\left(x_{1}\right) \Gamma_{j}\left(x_{2}\right) F_{j}^{i}(b)
$$

where $\Gamma_{i}(x)$ the usual one body parton distribution is function and $F_{j}^{i}(b)$ is a function normalized to one and representing the pair density in transverse space. The inclusive cross section hence simplifies to:

$$
\sigma_{(A, B)}^{D}=\frac{m}{2} \sum_{i j k l} \Theta_{k l}^{i j} \hat{\sigma}_{i j}(A) \hat{\sigma}_{k l}(B),
$$

where $\hat{\sigma}_{i j}(A)$ and $\hat{\sigma}_{k l}(B)$ are the hadronic inclusive cross section for the two partons labeled $i$ and $j$ undergo the hard interaction labeled $A$ and for two partons $k$ and $l$ to undergo the hard interaction labeled $B$;

$$
\Theta_{k l}^{i j}=\int \mathrm{d}^{2} b F_{k}^{i}(b) F_{l}^{j}(b)
$$

Are the geometrical coefficients with dimension an inverse cross section and depending on various parton processes. These coefficients are the experimentally accessible quantities carrying the information of the parton Correlation in transverse momentum. The cross section for multiple parton collisions has been further simplified as:

$$
\sigma_{(A, B)}^{D}=\frac{m}{2} \frac{\hat{\sigma}(A) \hat{\sigma}(B)}{\sigma_{e f f}}
$$

where all the information on the structure of the hadron in transverse space is summarized in the value of the scale factor. The experimental value measured by CDF yields $[19,20] \quad \sigma_{\text {eff }}=14.5 \pm 1.7_{-2.3}^{+1.7} \mathrm{mb}$. It is believed that is largely independent of the center-of-mass energy of the collision and on the nature of the partonic interactions. The experimental evidence is not inconsistent with the simplest hypothesis of neglecting correlations in momentum fractions.

\section{Results for $b \bar{b} h$ Production}

We evaluate the fully cross section for $b \bar{b} h$ production by requiring that the transverse momentum of both final state bottom and anti-bottom quarks be larger than 20 $\mathrm{GeV}$. This corresponds to an experiment measuring the 
Higgs decay products along with two high $p t$ bottom quark jets. These cuts reduce the cross section by one or two orders of magnitude, but also greatly reduce the background and ensure that the Higgs boson was emitted from a bottom quark and is therefore proportional to the square of the $b$-quark Yakawa coupling.

The cross section for leading order sub-process for Higgs-boson production in association with bottom quarks obtained using MRST parton distribution [21,22], the packages MadGraph [24] and HELAS [25] and the integration was performed by VEGAS [25] as function of Higgs mass for the LHC with $\sqrt{s}=14 \mathrm{TeV}$ are displayed in Figure 2.

The cross-section for Higgs production in association with bottom quarks are not large but may be useful if high luminosity is available, since the Higgs boson can be "tagged" by trigging on the bottom quarks.

Sizable rates of events where two bottom quarks associate with Higgs boson are produced contemporarily at the LHC, as a consequence of the large parton luminosity. The corresponding integrated rate is evaluated by combining the integrated cross section for Higgs boson and $b \bar{b}$ production at LHC energy.

If one uses the cross section for Higgs boson production from $p p \rightarrow H+X$, and the value for the scale factor $\sigma(b \bar{b}) \cong 5 \times 10^{2} \mu \mathrm{b} \quad$ (the observed value is

$\sigma_{\text {eff }}=14.5 \pm 1.7_{-2.3}^{+1.7} \mathrm{mb}$ one obtains the cross section for a double parton collision producing a Higgs boson and a $b \bar{b}$ pair.

The large rate of $b \bar{b}$ pair at the LHC gives rise to a relatively sizable production of Higgs boson associated with $b \bar{b}$ quarks.

Figure 3 shows the double parton scattering to the Higgs boson associated with $b \bar{b}$ quarks is very near in value to single parton scattering mechanism, so that we

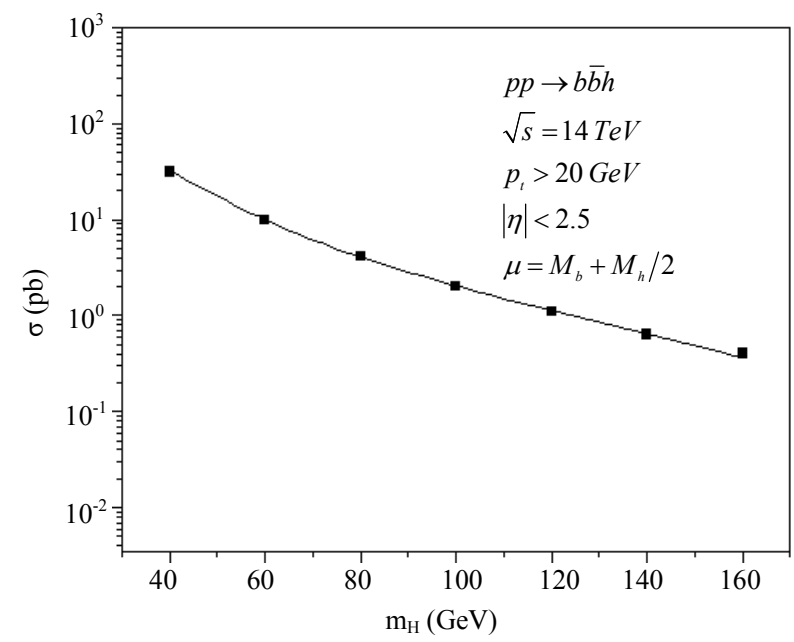

Figure 2. Leading order section (pb) for Higgs boson production in association with bottom quarks at the LHC.

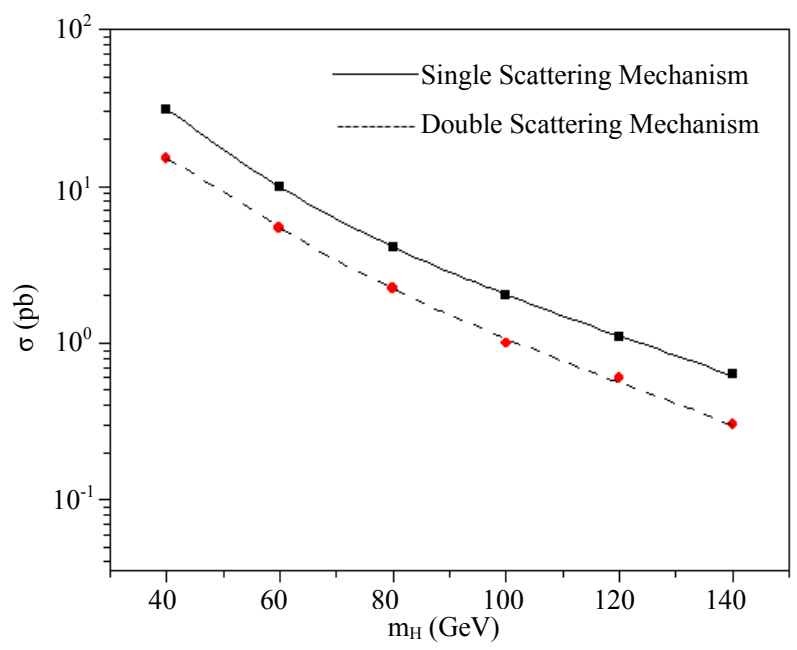

Figure 3. Total cross section for Higgs boson assocoated with bottom quarks in the SM at LHC energy.

have to take this in our consideration in any kind of search for Higgs discovery.

\section{Results for $t \overline{t h}$ Production}

The possibility of discovering a Higgs boson in the range $115-130 \mathrm{GeV}$ is becoming increasingly likely. The Standard Model precision fits are consistent with a light boson. Both the Fermilab and the CERN Large Hadron Collider will focus on the search of light Higgs boson, below the W-pair threshold, a Higgs boson mainly decays hadronically into $b \bar{b}$ pairs.

The associated production of a Higgs with a pair of $t \bar{t}$ quarks has drawn increasing attention. In spite of the very small cross section, this production mode has an extremely distinctive signature, and recent analyses have shown that it can be within the reach of the LHC. This process will provide a direct measurement of the topquark Yukawa coupling and will be instrumental in determining ratios of Higgs couplings in a model independent way.

Our calculation are found using MRST parton distribution [24], the packages MadGraph [25] and HELAS [26] and the integration was performed by VEGAS [27] as function of Higgs mass for the LHC with $\sqrt{s}=14 \mathrm{TeV}$ are displayed in Figure 4.

If one uses the cross section for Higgs boson production from $p p \rightarrow H+X, \sigma(t \bar{t}) \cong 850 \mathrm{pb}$ as a value for the scale factor (the observed value is) one obtains the cross section for a double parton collision producing a Higgs boson and a $t \bar{t}$ pair.

The small rate of $t \bar{t}$ pair with Higgs boson at the LHC gives rise to a relatively very small value for the production of Higgs boson associated with $t \bar{t}$ quarks.

Figure 5 shows the double parton scattering to the Higgs boson associated with $t \bar{t}$ quarks. Althought the 


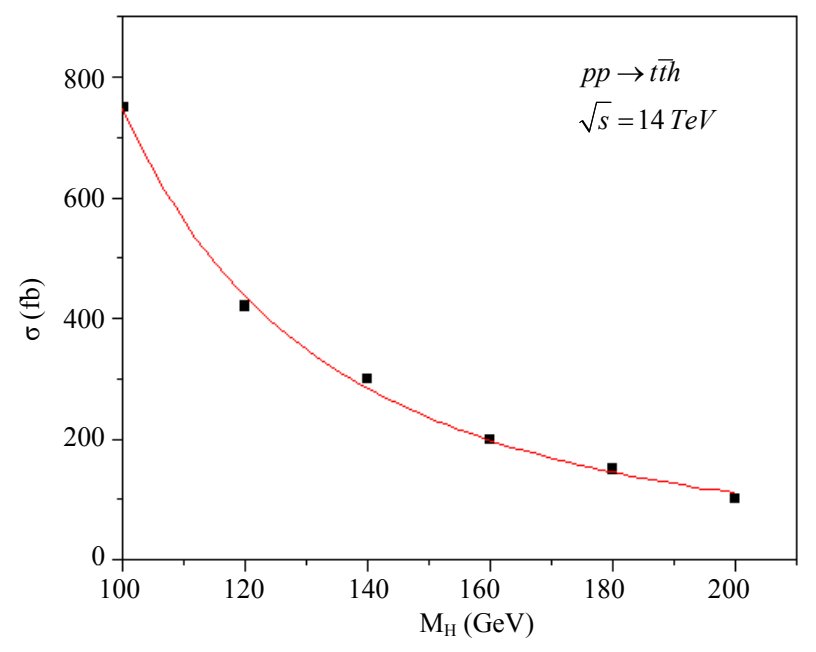

Figure 4. Leading order section (pb) for Higgs boson production in association with top quarks at the LHC.

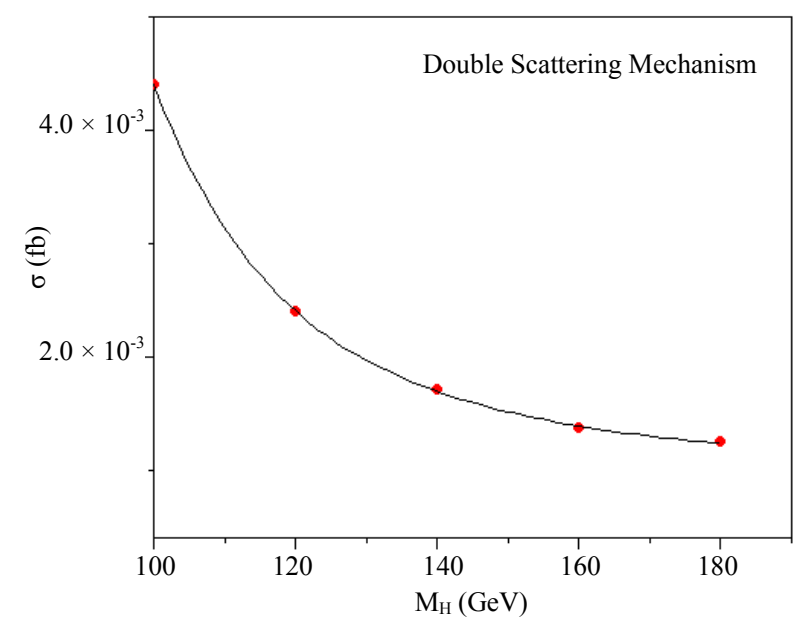

Figure 5. Total cross section for Higgs boson production with top quarks in the double parton scattering at the LHC.

rate of production cross section in double parton scattering mechanism is rather very small comparing with the rate of production in single parton scattering, but still in case of any change in the center of mass energy or luminosity of the collider may the effect be more clear in any calculation for the search of Higgs boson.

\section{Conclusions}

In this work we have investigated $b \bar{b} h$ production at the LHC, which is important discovery channel for Higgs boson in the SM and its extension in the MSSM at large values of $b \bar{b} h$, where the bottom Yukawa coupling is strongly enhanced.

Our calculations correspond to the cross section for Higgs boson in association with two tagged $b$ jets in single and double parton scattering mechanism.

Although the double parton collision cross section is not large, but it should be taken in consideration because a sizable rate of events where pairs of quarks are produced at the LHC, as a consequence of the large parton luminosity.

The technique used to calculate the cross section for $p p \rightarrow b \bar{b} h$ is also applied to the study of the associated production of $t \overline{t h}$. The cross section in double parton scattering mechanism for $t \overline{t h}$ case is very small and will probably be beyond the LHC machine capabilities.

However, in the hadron collider environment, the large QCD backgrounds may cause the observation impossible for those events if Higgs bosons decay hadronically. Individual channels with hadronic decays should be studied on case by case.

\section{REFERENCES}

[1] T. Han and S. Willenbrock, "QCD Corrections to the $p p \rightarrow \mathrm{WH}$ and ZH cross Sections," Physical Letters B, Vol. 273, 1991, p. 167.

[2] T. Han, G. Valencia and S. Willenbrock, "Structure Function Approach to Vector Boson Scattering in $p p$ Scattering," Physical Review Letters, Vol. 69, 1992, p. 3274.

[3] S. Dittmaier, M. Kramer and M. Spira, "Higgs Radiation off Bottom Quarks at the Tevatron and the CERN LHC," Physical Review D, Vol. 70, 2004, Article ID: 0704010.

[4] S. Dawsen, C. B. Jakson, L. Rena and D. Wackeroth, "Exclusive Higgs Boson Production with Bottom Quarks at Hadron Colliders," Physical Review D, 69, 2004, Article ID: 074027.

[5] S. Dawson, "Radiative Corrections to Higgs Boson Production," Nuclear Physics B, Vol. 359, No. 2-3, 1991, pp. 283-300. doi:10.1016/0550-3213(91)90061-2

[6] A. Djouadi, M. Spira and P. M. Zerwas, "Production of Higgs Bosons in Proton Colliders. QCD Corrections," Physical Letters B, Vol. 264, No. 3-4, 1991, pp. 440-446. doi:10.1016/0370-2693(91)90375-Z

[7] D. Grandenz, M. Spira, and P. M. Zerwas, "QCD Corrections to Higgs Boson Production at Proton Colliders," Physical Review Letters, 70, 1993, p. 1472.

[8] M. Spira, A. Djouadi, D. Grandenz and P. M. Zerwas, "Production of Higgs Bosons in Proton Colliders. QCD Corrections," Physical Letters B, Vol. 264, No. 3-4, 1991, pp. 440-446. doi:10.1016/0370-2693(91)90375-Z

[9] G. Aad, et al., "Observation of a New Particle in the Search for the Standard Model Higgs Boson with the ATLAS Detector at the LHC," Physical Letters B, Vol. 716, No. 1, 2012, pp. 1-29. doi:10.1016/j.physletb.2012.08.020

[10] S. Chatrchyan, et al., "Observation of a New Boson at a Mass of $125 \mathrm{GeV}$ with the CMS Experiment at the LHC," Physical Letters B, Vol. 716, No. 1, 2012, pp. 30-61. doi:10.1016/j.physletb.2012.08.021

[11] S. Moch and M. Vogt, "Higher-Order Soft Corrections to Lepton Pair and Higgs Boson Production," Physical Letters $B$, Vol. 631, 2005, p. 48. 
[12] E. Laenen and L. Mugnea, "Threshold Resummation for Electroweak Annihilation from DIS Data," Physical Letters B, Vol. 632, 2005, p. 270.

[13] T. Han, G. Valencia and S. Willenbrock, "Structure Function Approach to Vector Boson Scattering in $p p$ Collision," Physical Review Letters, Vol. 69, 1992, p. 3274.

[14] E. Berger and J. Campbell, "Higgs Boson Production in Weak Gusion at Next-to-Leading Order," Physical Review D, Vol. 70, 2004, Article ID: 073011.

[15] J. Campell, R. Ellis, F. Maltoni and S. Willenbrock, "Higgs Boson Production in Association with a Single Bottom Quark," Physical Review D, Vol. 67, 2003, article ID: 095002.

[16] S. Dawson, C. B. Jackson, L. Reina and D. Wackeroth, "Higgs Boson Production with Bottom Quarks at Hadron Colliders," International Journal of Modern Physics A, Vol. 20, No. 15, 2005, p. 3353. doi: $10.1142 / \mathrm{S} 0217751 \mathrm{X} 05026558$

[17] Dawson, C. B. Jackson, L. H. Orr, L. Reine and D. Wackeroth, "Theoretical Progress for the Associated Production of a Higgs Boson with Heavy Quarks at Hadron Colliders," European Journal of Physics C, Vol. 33, 2004, p. 5451.

[18] J. Campbell, S. Dawson, S. Dittmaier, C. Jackson, M. Kramer, F. Maltani, L. Reina, M. Spira, D. Wackeroth and S. Willenbrock, "Higgs Boson Production in Association with Bottom Quarks," hep-ph/0405302.

[19] C. Goebel, F. Halzen and D. H. Scott, "Double Drell-Yan Annihilation in Hadron Collisions: Novel Tests of the Constituents Picture," Physical Review D, Vol. 22, 1980, pp. 2789.
[20] F. Halzen, P. Hoyer and W. J. Striling, "Evidence for Multipe Parton Interaction from the Observation of Multi-Muon Events in Drell-Yan Experiment," Physical Letters B, Vol. 188, 1987, pp. 375.

[21] N. Paver and D. Treleani, "Multiquark Scattering and Large- $p_{\mathrm{T}}$ Jet Production in Hadronic Collisions," Nuovo Cimento A, Vol. 70, No. 3, 1982, pp. 215-228. doi: $10.1007 / \mathrm{BF} 02814035$

[22] M. Broun and D. Treleani, "The Double Parton Distributions in the Hard Pomeron Model," European Journal of Physic C, Vol. 18, No. 3, 2001, p. 511-522. doi:10.1007/s100520100565

[23] F. Abe, et al., "Double Parton Scattering in $\overline{p p}$ Collisions at $\sqrt{s}=1.8 \mathrm{TeV}$," Physical Review D, Vol. 56, No. 7, 1997, pp. 3811-3832. doi:10.1103/PhysRevD.56.3811

[24] A. D. Martin, R. G. Roberts, W. J. Stirling and R. S. Thorne, "Parton Distribution at the LHC: W and Z Production," European Journal of Physic C, Vol. 14, 2000, p. 133.

[25] T. Stelzer and W. F. Long, "Automatic Generation of Tree Level Helicity Amplitudes," Computation Physical Communications, Vol. 81, 1994, pp. 337.

[26] E. Murayama, I. Watanabe and K. Hagiwara, "HELAS: Helicity Amplitude Subroutine for Feynman Diagram Evaluations," KEK Report, 1992, p. 91.

[27] G. P. Lepage, “A New Algorithm for Adaptive Multidimensional Integration," Journal of Computation Physics, Vol. 27, No. 2, 1978, pp. 192-203. doi:10.1016/0021-9991(78)90004-9 